\title{
Le genre du texte, un outil didactique pour développer le langage oral et écrit
}

Joaquim Dolz et Roxane Gagnon

\section{(2) OpenEdition}

1 Journals

\section{Édition électronique}

URL : https://journals.openedition.org/pratiques/1159

DOI : 10.4000/pratiques.1159

ISSN : 2425-2042

Éditeur

Centre de recherche sur les médiations (CREM)

\section{Édition imprimée}

Date de publication : 15 juin 2008

Pagination : 179-198

\section{Référence électronique}

Joaquim Dolz et Roxane Gagnon, « Le genre du texte, un outil didactique pour développer le langage oral et écrit », Pratiques [En ligne], 137-138 | 2008, mis en ligne le 15 juin 2008, consulté le 01 octobre 2021. URL : http://journals.openedition.org/pratiques/1159; DOI : https://doi.org/10.4000/pratiques. 1159

\section{(c) Tous droits réservés}




\section{Le genre du texte, un outil didactique pour développer le langage oral et écrit}

\section{Joaquim Dolz et Roxane Gagnon}

Faculté de psychologie et des sciences de l'éducation Université de Genève

Cette contribution cherche à rendre hommage à Jean-François Halté en poursuivant le débat scientifique, entamé depuis la publication de son ouvrage La didactique du français (1992), au regard de l'oral et de son enseignement. Reprenant les propositions d'Halté (1993) au sujet de ce qu'il appelait le « serpent de mer » ${ }^{(1)}$, nous référant principalement à l'article « Intégrer l'oral : Pour une didactique de l'activité langagière » (2005), nous discuterons trois thèmes développés dans ses écrits :

- l'oral comme objet d'enseignement : peut-on enseigner l'oral ? si oui, quel oral enseigner? comment faire de l'oral un objet enseignable ?

- l'intégration des rapports oral/écrit dans l'enseignement : comment envisager la continuité entre l'oral et l'écrit?

- les dimensions didactiques et pédagogiques liées à l'enseignement-apprentissage de l'oral : comment articuler l'oral à enseigner des didactiques et l'oral outil pour apprendre des pédagogues?

Pour développer notre position, nous présenterons, à la suite des travaux de l'équipe genevoise de didactique des langues (Dolz \& Schneuwly, 1998), la notion de genre et expliciterons son rôle dans l'enseignement et l'apprentissage de la production textuelle à l'oral comme à l'écrit.

Dans un premier temps, nous présenterons la notion de façon générale, en posant notamment quelques jalons historiques qui permettent de comprendre son évolution et sa signification actuelle. Nous décrirons ensuite le point de vue communicationnel tel que théorisé par Bakhtine (1984), vu sa pertinence. Puis, nous expliciterons comment il est possible d'analyser le genre comme méga-outil didactique (Schneuwly, 1994 ; Dolz, Moro \& Pollo, 2000). Dans un deuxième temps, nous montrerons en quoi et comment la notion de genre contribue à l'en-

(1) Halté qualifiait l'oral ainsi pour «ses grandes capacités en apnée et son attitude à ressurgir périodiquement de l'océan des préoccupations éducatives » (Halté, 2005, p. 11) 
seignement-apprentissage de la production textuelle, adoptant le point de vue de la didactique des langues. En outre, nous poserons le genre comme objet d'enseignement-apprentissage et présenterons son modèle didactique, lequel génère des séquences didactiques. La troisième partie de cet article vise à illustrer la notion de genre et son usage didactique par un exemple précis. Nous pointerons les potentialités de travailler l'improvisation théâtrale, genre oral, pour l'écriture d'une saynète. Ce cas particulier nous servira à souligner la dynamique inhérente aux genres textuels, dynamique qui en fait des outils constamment actualisés, apportant une réponse pertinente et cohérente aux défis de l'enseignement. En conclusion, nous reviendrons aux trois thèmes chers à Halté et dirons en quoi nos positions se dissocient ou s'associent à celles du regretté didacticien.

\section{Le genre : du classement des textes à l'outil d'enseignement-apprentissage}

La notion de genre a déjà fait couler beaucoup d'encre, nous ne pouvons donc prétendre en aucun cas à l'exhaustivité. De nombreux ouvrages reconnus sont parus sur le sujet et nous ne pouvons qu'en lister les principaux. Une synthèse de la notion de "genres littéraires » a été faite par Schaeffer en 1989, par Combe en 1992 et, plus particulièrement dans le cadre de l'enseignement de la littérature, par Canvat en 1999. Nous renvoyons le lecteur, pour l'analyse sémantique de la notion, aux Genres du discours de Todorov et à l'Introduction à l'architexte de Genette, parus respectivement en 1978 et en 1979. Fowler (1982) a fait paraitre ${ }^{(2)}$ un ouvrage sur la généricité et sur le statut des catégories génériques, intitulé Kinds of Literature. On doit à Jauss la notion « d'horizon d'attente générique " $(1975 / 1978)$ qui instaure une théorie de la réception des textes littéraires. Soulignons aussi deux ouvrages, s'inscrivant dans une réflexion sur les typologies à laquelle ont pris part de nombreux chercheurs de Suisse romande : Les textes, types et prototypes (1992) du linguiste J.-M. Adam et, en psychologie du langage, Activité langagière, textes et discours (1996) de J.-P. Bronckart. Un survol des ouvrages existant sur la notion permet toutefois de s'apercevoir que l'optique de la production demeure une avenue peu fréquentée, ce qui est tout à notre honneur puisque nous lui consacrerons les pages qui suivent. Plus particulièrement, nous nous intéressons aux genres comme objets d'enseignement-apprentissage de la production écrite et orale, partant du postulat que ce sont les pratiques langagières signifiantes, socialement reconnues qui doivent orienter l'enseignement.

\section{Essai de définition de la notion}

Tout texte est singulier, il est l'œuvre d'un individu ou d'un collectif à un moment donné. Or, cet acte de rédaction, construction de sens, s'inscrit dans une époque, dans une culture, s'écrit dans une langue et selon une visée particulière. Dans la perspective historico-culturelle ${ }^{(3)}$ qui est la nôtre, nous croyons à l'instar de Bronckart (1996) que tout membre d'une communauté est confronté à un

(2) Nous appliquons les rectifications orthographiques de 1990.

(3) Notre perspective est aussi fondamentalement sociale, mais nous tenons ici à souligner les dimensions historiques cristallisées au cours de pratiques culturelles effectives. Souligner l'inscription de ces pratiques dans une continuité. 
univers de textes « déjà là », univers organisé en "genres » empiriques et historiques, c'est-à-dire en forme d'organisations concrètes qui se modifient avec le temps. Socialement et culturellement, tout texte est donc un exemplaire de genre. Molière, écrivant Les Précieuses Ridicules, rédige les dialogues des personnages, dialogues qui construisent l'action d'une des scènes de cette pièce de théâtre inédite. Celle-ci, savant mélange entre texte dramatique de style soutenu, ornements musicaux et chorégraphies, oscillant entre la farce et la satire, est l'un des coups d'éclat qui permet à l'auteur de conquérir la cour et le roi. L'exemple de la pièce de Molière illustre le caractère dynamique des genres : sortes d'entités collectives à parentés multiples s'inscrivant dans un horizon d'attentes générique qu'ils déplacent et réorganisent (Schaeffer, 2001).

Définir la notion de genre demande d'abord de considérer l'ancrage social et la nature communicationnelle du discours. Prenons l'exemple d'un genre tel que l'entretien d'embauche en vue de présenter sa candidature pour un emploi, la prise en compte des dimensions de la situation d'interaction sont fondamentales dans la compréhension du genre, à savoir l'adaptation au lieu de travail, au destinataire et au profil du poste convoité ainsi que la prise en compte de la visée, mettre en valeur sa candidature pour qu'elle soit sélectionnée parmi les autres candidatures.

En plus du contexte social, il y a à considérer les régularités compositionnelles et les caractéristiques formelles des textes produits (Charaudeau \& Maingueneau, 2002). Ces régularités correspondent, en d'autres termes, au cadre pragmatique dans lequel tout texte s'inscrit. Ce cadre est contraignant, puisqu'il est régi par des normes (Schaeffer, 2001) et que celles-ci présupposent des choix langagiers, linguistiques et communicationnels. Il s'en dégage des catégories (Chareaudeau \& Maingueneau, 2002) qui stabilisent des formes d'association entre des formes d'action (rôles discursifs, tâches cognitives), des contenus et des manières de dire (dispositifs d'énonciation, nouvelles dénominations, apparition de formules permettant de ritualiser les pratiques, etc.). Si nous poursuivons notre exemple, l'entretien d'embauche prend place dans une situation relativement réglée du point de vue des conventions sociales (rituels d'ouverture et de clôture, postures énonciatives en fonction du poste convoité, attitudes corporelles, formules et gestes marquant la politesse, etc.). Il est par ailleurs généralement précédé par une lettre de motivation, genre écrit, dont les régularités permettent leur reconnaissance dans une culture. Les contenus de l'entretien d'embauche permettent d'intégrer et d'expliciter ceux de la lettre de motivation.

On désigne les genres selon les sphères d'activités, ils se différencient donc en fonction de leur but dans chacune de ces sphères (les médias, l'école, la politique, l'Église, etc.). Des distinctions existent aussi selon les cultures et selon les langues : à l'écrit, un éditorial de presse dans la tradition espagnole diffère de l'éditorial de la presse francophone ${ }^{(4)}$; à l'oral, les genres oraux du griot mandingue comme la palabre ou le « baro » (Toulou, 2005) ne connaissent d'équivalent dans la culture française. Le fait qu'un texte singulier présente des caractéristiques communes à d'autres textes produits dans des situations similaires permet son repérage comme exemplaire d'un genre. Pour revenir à notre exemple, les contenus d'un entretien d'embauche se rapportent aux compétences professionnelles et à la mise en valeur de celles-ci par le candidat. L'organisation de la

(4) L'éditorial en Espagne n'est pas signé, il est la voix du comité éditorial. Un article d'opinion signé n'y est pas considéré comme un éditorial. 
lettre suit un plan associé à la forme conventionnelle du genre, l'étayage argumentatif de la valeur de la candidature se présente selon une structure hiérarchique spécifique.

Enfin, du point de vue linguistique, l'entretien d'embauche contient de nombreuses formules d'usage pour l'établissement du contact et la démonstration de politesse avec une phraséologie caractéristique, des marques modales pour nuancer les prises en charge énonciative et des organisateurs logico-argumentatifs pour ordonner les arguments, etc. C'est un ensemble d'unités linguistiques, mises en relation de co-textualité particulière, qui permet d'identifier un texte comme appartenant à un genre et qui caractérise les formes linguistiques spécifiques de sa textualité.

Bakhtine (1984) avait déjà défini le genre textuel comme la fusion de trois dimensions essentielles : des contenus devenus dicibles à travers lui, la structure communicative particulière des textes, des configurations spécifiques d'unités langagières, traces de la position énonciative et des ensembles de séquences textuelles et de types discursifs qui forment la structure du genre.

Dans la tradition littéraire, les genres permettent le regroupement des différents textes du patrimoine culturel pour leur étude. Or, les critères dont découle cet assemblage posent problème puisqu'à travers les siècles, ils varient énormément, se rapportant à la forme, au contenu, aux différentes façons de représenter le réel, liés à une école ou une période historique spécifique, à l'organisation énonciative (Charaudeau \& Maingueneau, 2002). Nous retraçons brièvement l'historique de la notion de genre, de façon à mieux en saisir le sens et la portée.

\section{Bref éclairage historique de la notion de genre}

L'élaboration conceptuelle de la notion de genre remonterait à l'Antiquité. Aristote s'intéresse aux transformations qui s'opèrent dans le langage pour que celui-ci, de simple outil de communication, devienne moyen de création. Le philosophe grec soutient qu'il ne peut y avoir de création, poièsis, que s'il y a mimèsis, représentation, simulation d'actions. Il distingue donc le fond et la forme. Ainsi, ce qui fait le poète, ce n'est pas la forme verbale (versifiée), mais la fiction. Aristote, répartissant les œuvres en fonction des objets représentés, distingue les modalités de représentation ${ }^{(5)}$ qu'il reprend de Platon, et les moyens utilisés. Il caractérise trois genres de discours : le judiciaire, l'épidictique et le délibératif. C'est pendant la période de l'Antiquité que les grands genres sont fixés et établis : épopée, élégie, tragédie, comédie, parodie, roman ${ }^{(6)}$, conte, fable, maxime, loi, épitaphe, oracle, ode, poème lyrique, etc.

Comme la culture antique, la culture du Moyen Âge est dominée par l'oralité ; elle voit apparaitre les chansons de geste, les fabliaux, les farces, les «mystères », les refrains, les comptines, les berceuses et les ballades. On y pratique aussi le brocard, adage juridique, les hymnes, le lyrisme, le théâtre " catharsis ", le roman, le récit historique (estoire), le texte didactique, le sermon, la lettre. Le développement de la « littérature d'imagination » conduit à ce qu'une distinction s'opère entre la « Première rhétorique», portant sur l'éloquence, et la «Se-

(5) Aristote répertorie trois niveaux de style : le familier, le tempéré et le sublime.

(6) Nous faisons référence ici au roman grec, tel qu'il se présente chez des auteurs comme Xénophon d'Ephèse, Diogène, Lucien et Chion (Loicq-Berger, 1980). 
conde rhétorique », recherchant l'ornementation du discours, cousinant avec la poétique (Canvat, 1999).

C'est au cours de la Renaissance que l'habitude de grouper les «textes » par genres se répand, ceux-ci acquièrent une fonction classificatoire. L'admiration des Anciens engendre une série d'emprunts qui permet, d'abord, le renouvellement et la création de genres, avec l'œuvre de la Pléiade, et, plus tard, la fixation de genres devenus des modèles, des sortes d'archétypes universels. Le classicisme, sous l'inspiration de Boileau et de Descartes notamment, renforce cette tendance en établissant des règles au nom de la raison : la règle des trois unités pour la tragédie classique, le souci de la bienséance, la sublimité du style.

Avec le romantisme, les genres reprennent un sens suivant l'évolution de la société et de la culture ; 1'art authentique n'est plus une imitation de la nature, mais l'expression d'un individu au-dessus du commun. Avec des penseurs comme Kant, le romantisme allemand affranchit l'artiste de sa dépendance à l'égard de la $m i$ mésis. Les frères Schlegel montrent l'historicité de la notion de genre et distinguent les formes subjectives des formes objectives. Les genres poétiques deviennent les genres par excellence, ce qui ouvre la voie à « la désintégration » (Canvat, 1999) des distinctions de genres. Pour les auteurs, il importe surtout de transgresser les frontières entre les genres. En France, Baudelaire brille par le poème en prose ; Hugo, avec Cromwell notamment, mélange l'ode, l'épopée et le drame.

A la fin du XIX ${ }^{\mathrm{e}}$ siècle, en raison de la diffusion de la presse et du poids croissant des contraintes économiques sur la production littéraire, on assiste à la réorganisation de la hiérarchie des genres et des valeurs littéraires. On cherche l'innovation, la singularité afin de ne pas véhiculer les stéréotypes typiques de la « littérature industrielle» (Canvat, 1999). Les attaques les plus directes à la théorie des genres vont venir toutefois des surréalistes qui s'efforcent par leurs trouvailles théoriques et pratiques d'accélérer la «désintégration» des genres.

Bakhtine, que l'on « découvre » en France en $1963^{(7)}$, en grande partie grâce aux travaux de Todorov et de Kristeva, s'intéresse au genre romanesque et tente d'en montrer notamment la multiciplicité des aspects, les principes fondamentaux de la structuration du héros romanesque, les emprunts, les transgressions.

Le roman parodie les autres genres (justement en tant que genres); il dénonce leurs formes et leurs langages conventionnels, élimine les uns, en intègre d'autres dans sa propre structure en les réinterprétant, en leur donnant une autre résonance. Les historiens de la littérature sont parfois enclins à n'y voir qu'une lutte de courants et d'écoles littéraires. Bien sûr, cette lutte existe, mais c'est un épiphénomène historiquement infime. Il faut savoir apercevoir au-delà un conflit des genres plus profonds, plus historique, un devenir et une croissance de l'ossature des genres littéraires (Bakhtine, 1978, p. 443).

Les notions de « dialogisme», de «polyphonie » et d' " intertextualité », telles que développées par le théoricien russe ${ }^{(8)}$, permettent la réhabilitation de la notion de genre dans le monde littéraire. Notion qui, après tout, demeure incontournable : comment vouloir transgresser les normes en niant leur existence ? L'opposition, la différenciation supposent une normalité, une similarité. Cela

(7) C'est en 1963 que fut réédité son ouvrage sur Dostoïevski, paru initialement en 1929.

(8) Mikhaïl Bakhtine a repris et transformés les thèmes développés par Voloshinov, pour une analyse fine, voir Bota \& Bronckart (2007). 
dit, le souci de déconstruction des postmodernes contribue à la notion de genre en lui apportant une certaine distance critique.

L'histoire de la notion et le renouvellement constant des genres, lié à celui des pratiques sociales, empêchent l'établissement à priori de typologies. Les genres textuels s'appréhendent dans des mouvements de rupture et de continuité. La dynamique inhérente à la notion de genre a été bien mise en lumière par le théoricien $\mathrm{Mi}$ kaël Bakhtine. C'est à son modèle théorique que nous nous arrêtons maintenant.

\section{L'apport de Bakhtine}

Le théoricien russe Mikhaïl Bakhtine (1895-1975) a voulu développer les bases d'une nouvelle linguistique, d'une « translinguistique », dont l'objet n'est plus l'énoncé mais l'énonciation et l'interaction verbale (Todorov, 1984). Pour lui, les énoncés, matérialisation de l'utilisation de la langue, émanent des représentations d'un domaine de l'activité humaine, ils sont issus de la fusion d'un contenu thématique, d'un style et d'une construction compositionnelle. Chaque sphère d'utilisation de la langue élabore ses types relativement stables d'énoncés, ces types stables constituent les genres du discours. Le genre fonctionne donc comme une norme qui intervient dans la structuration des énoncés, comme le font les formes du langage. Bakhtine (1984) souligne le caractère mouvant de ces genres :

La richesse et la variété des genres du discours sont infinies, car la variété virtuelle de l'activité humaine est inépuisable et chaque sphère de cette activité comporte un répertoire des genres du discours qui va se différenciant et s'amplifiant à mesure que se développe et se complexifie la sphère donnée (p. 265).

Cette variété, cette richesse se lient à la dynamique existant entre les genres premiers et les genres seconds. Bakhtine distingue les genres premiers, ceux des échanges verbaux ordinaires, et les genres seconds, issus du discours littéraire, scientifique ou idéologique, nés d'échanges culturels plus complexes et plus poussés. Les genres seconds absorbent et transmutent les genres premiers, mais cessent d'avoir un rapport immédiat au réel existant et aux énoncés d'autrui (Bakhtine, 1984).

L'interrelation entre les genres premiers et seconds d'une part, le processus historique de formation des genres seconds d'autre part, voilà qui éclaire la nature de l'énoncé (et, tout particulièrement, le problème difficile de la corrélation entre langue, idéologies et visions du monde) (Bakhtine, 1984, pp. 267-268).

Illustrons l'interrelation entre genres seconds et genres premiers par l'exemple de la recette de cuisine. La recette enseignée par une mère à sa fille, dans la cuisine familiale, n'est pas celle de l'animatrice vedette à la télévision. Même si, dans les deux cas, il peut y avoir un rapport immédiat au réel existant, le niveau de spontanéité et la quotidienneté des échanges entre la mère et la fille appartiennent à ce que Bakhtine nomme les genres premiers. L' «émission de cuisine » est, pour des raisons historiques, commerciales et artistiques, en grande partie prévue et pensée, donc médiatisée. Bien que ce type d'émission s'inspire des pratiques du quotidien, son énonciateur s'adresse à un public anonyme. La recette du livre de cuisine appartient également aux genres seconds : écrite, elle n'a pas ce rapport immédiat au réel et, au plan de la forme et de la structuration du contenu, elle présente les informations de façon plus condensée et en respectant une succession. Les genres, une fois médiatisés, forment des entités intermédiaires qui 
stabilisent les éléments formels et les rituels des pratiques (de Pietro \& Dolz, 1997). Par exemple, la scolarisation des genres entraine des transformations, nous verrons plus loin que celles-ci sont faites de façon consciente et inconsciente par les partenaires de l'enseignement.

Bakhtine instaure la notion de dialogisme des échanges langagiers qui soustend que tout énoncé s'appréhende dans sa tension, son orientation vers autrui. Cette position présuppose que l'objet de l'énoncé ou du discours se fonde sur des représentations supposées partagées qui permettent un ajustement des systèmes de repérage du locuteur et du destinataire (Canvat, 1999). Les genres sont des outils qui instituent la possibilité de communication (Schneuwly, 1994).

\section{Le genre comme outil d'enseignement-apprentissage}

Le terme outil peut référer au moyen culturel élaboré par l'homme pour transformer la nature. Marx (1968) considère d'ailleurs que l'un des traits caractéristiques de l'humain est d'être un « animal fabricateur d'outils» (p. 182). Ces outils fabriqués par l'homme se qualifient de «culturels » puisqu'ils s'inspirent des pratiques de l'homme et se transmettent d'une génération à l'autre. Leur transmission est en grande partie assurée par l'éducation. Les outils techniques permettent aux hommes de contrôler la nature extérieure, mais pour contrôler les comportements humains, ceux d'autrui comme leurs propres comportements, les humains ont créé des outils spécifiques que Vygotski (1930/1985) désigne par symboles ou outils psychologiques. Il est donc possible de transposer le concept d'outil à la réalité psychique humaine, guidée en cela par l'idée générale que la transformation et la maitrise des processus psychiques nécessitent des outils mentaux. Suivant les théories de Vygotski, nous concevons le développement humain comme une adaptation artificielle, médiatisée par des outils psychiques, transformant fondamentalement les capacités psychiques, celles-ci existant d'abord sous forme excentrée dans les produits de la société humaine, telles un déjà-là devant être investi de significations (Dolz, Moro, Pollo, 2000). L'outillage mental de l'élève est un bagage de techniques, de savoir-faire et de connaissances accumulées au cours des siècles et transmis à l'enfant par son entourage (Alcorta, 2001).

Le développement ne saurait se réduire à un mouvement de complexification des structures initiales. Il réside au contraire dans le processus toujours original par lequel cet être de nature qu'est le jeune enfant s'approprie au sein de situations communicatives, les outils culturels les plus élaborés de son temps et se trouve transformé du fait même de cette appropriation (Brossard, 2001, p. 53).

Le développement consiste en l'appropriation des outils initialement excentrés dans leurs usages, en une (re)construction progressive de ce capital culturel initialement excentré (Schneuwly, 1994 ; Dolz, Moro, Pollo, 2000 ; Wirthner, 2006). L'articulation entre les outils et le développement se fait par des médiations éducatives (Dolz, Moro, Pollo, 2000). Aussi, par outil didactique, nous entendons, à l'instar de Plane et Schneuwly (2000), tout artéfact introduit dans la classe de français servant l'enseignement-apprentissage des notions et capacités mis au service d'un enseignement ou d'un apprentissage particulier.

Médiateurs essentiels de l'activité humaine, associés à des sphères d'activités spécifiques, les genres sont vus comme des outils pour agir dans des situations langagières ; leurs potentialités développementales s'actualisent et s'appro- 
prient dans l'usage. De plus, en raison de leur caractère matriciel et productif d'activités et d'action, il est possible de les considérer comme des méga-outils (Dolz, Moro, Pollo, 2000). Outils culturels, ils sont aussi des outils didactiques, à la fois outils d'enseignement et outils d'apprentissage. Comme outil d'enseignement, le genre fixe des significations sociales complexes concernant les activités d'apprentissages langagières. Il oriente la réalisation de l'action langagière, tant du point de vue des contenus, qui lui sont afférents et qui sont dicibles par lui, que du point de vue de la structure communicationnelle et des configurations d'unités linguistiques auxquelles il donne lieu (sa textualisation). Le genre apporte aussi un éclairage nouveau sur l'objet enseigné, il conduit l'enseignant à modifier sa façon de se représenter la production textuelle et son enseignementapprentissage (Wirthner, 2006). Quand il devient outil d'apprentissage, il permet à l'élève qui le pratique d'avoir accès à certaines de ses significations et, s'il les intériorise, d'accroitre ses capacités langagières. A l'heure actuelle, les relations entre le genre comme outil d'enseignement-apprentissage, transférable à d'autres disciplines, et le genre comme objet d'enseignement-apprentissage, faisant partie de la matrice disciplinaire du français, restent à être déterminées, tout comme les relations entre le genre outil et les autres outils de l'enseignant de français (Dolz, Schneuwly, Thevenaz-Christen, 2007).

\section{Enseigner et apprendre par les genres}

Du point de vue didactique, le texte est considéré depuis longtemps comme l'unité de base de l'enseignement de la production orale et écrite, mais aussi de la lecture et de la réception orale. Ce choix s'explique tout d'abord par des raisons associées aux situations de communication. Les textes correspondent à des situations d'interaction sociale, s'inscrivent dans un horizon social et s'adaptent à un auditoire particulier. La communication ne se produit pas au moyen de phrases ou de mots isolés, mais par le biais de textes oraux et écrits. Il ne suffit pas de faire des phrases correctes pour raconter un conte ou rédiger un roman. Dans une perspective interactionniste et sociodiscursive, il semble donc important d'insister sur les situations d'interaction sociale qui déterminent les activités langagières des apprenants et sur le fait que toute activité langagière mobilise des signes qui se matérialisent en textes. Le texte se présente comme l'unité de base de l'enseignement, en ce qu'il est à la fois unité fonctionnelle de la communication et unité attestable de l'activité langagière. Mais cette centration sur le texte n'est pas sans problèmes. Le premier problème a trait à la grande variété d'activités langagières. Le fonctionnement de la langue se caractérise par l'énorme diversité de pratiques textuelles qui varient en fonction des buts à atteindre, des situations et des conventions sociales. Pour l'enseignement, il y a à trouver des stratégies pour aborder cette diversité. La deuxième difficulté pour l'enseignement a trait au classement de ces textes puisque, comme nous l'avons vu, ceux-ci sont dynamiques, évoluent dans le temps et sont impossibles de classer de manière stable. Nous avons dû d'ailleurs procéder à un regroupement des genres textuels en fonction de critères didactiques (Dolz \& Schneuwly, 1998 ; Dolz, Noverraz \& Schneuwly, 2001). Cinq rubriques ont été créées : narrer, relater, transmettre des savoirs, argumenter et réguler des comportements. A ces cinq rubriques, il faut en ajouter ne autre, plus transversale, mettre la langue en jeu pour les textes poétiques. 


\section{Entrer par les genres dans l'enseignement de la langue}

La notion de genre s'avère centrale pour la construction de capacités langagières des apprenants. A ce propos, nous avons défendu la thèse que « c'est au travers des genres que les pratiques langagières s'incarnent dans les activités des apprenants » (Schneuwly \& Dolz, 1997, p. 29). Nous évoquerons ici rapidement les principaux avantages qu'une entrée par les genres présente.

Tout d'abord, devant la diversité et l'hétérogénéité des textes, le travail de regroupement facilite le déploiement de contenus d'enseignement. Comme il met l'accent sur les caractéristiques communes des genres regroupées dans la rubrique, il permet l'économie du travail de certaines composantes au cours de l'enseignement. Par exemple, le travail sur les caractéristiques communes des textes narratifs aidera à mettre en évidence les particularités d'un genre comme le récit d'énigme. Au moment d'aborder ce genre, on peut s'attendre à ce que certains transferts s'effectuent par rapport aux genres narratifs de fiction abordés précédemment. Ainsi, le travail sur la diversité suppose l'adoption d'une perspective différentielle et comparative, axée sur les apprentissages spécifiques et contrastés pour chaque genre.

Le deuxième avantage concerne les possibilités de prise en considération des pratiques sociales de référence. L'analyse de l'usage des genres constitue un référent pour évaluer la pertinence, l'adaptation et l'efficacité communicative des textes. Le didacticien a besoin de considérer le contexte social de production et de réception des textes. Par exemple, le recueil d'un corpus de débats semble indispensable pour connaitre les caractéristiques spécifiques de ce genre, mais aussi pour anticiper le traitement didactique en vue de l'adaptation aux capacités des élèves (Dolz \& Schneuwly, 1998).

Le troisième avantage renvoie aux aspects associés aux représentations "génériques » collectives véhiculées par l'usage du genre. Dès qu'on parle, par exemple, d'un constat d'accident, nous nous représentons presque immédiatement la situation dans laquelle ce texte se produit et ses caractéristiques matérielles et linguistiques. Les genres sont nommés, identifiés et catégorisés par les usages. Travailler à partir des représentations sociales facilite le « sens » des apprentissages. La représentation du genre fixe l'horizon d'attente pour le producteur et pour le récepteur. Le genre oriente non seulement les activités et les stratégies de lecture et d'écriture de l'apprenant, mais permet le développement de stratégies d'intervention organisée en fonction des connaissances existantes sur les stratégies utilisées par les lecteurs et les scripteurs débutants.

Comme nous l'avons déjà signalé, un genre est un préconstruit historique résultant d'une pratique et d'une formation sociale. L'apprentissage du langage oral et écrit se fait par la confrontation à un univers de textes « déjà là ». C'est une appropriation d'expériences accumulées par la société. L'enseignement scolaire s'organise de ce point de vue dans une perspective historique et culturelle. Il s'agit de contribuer à construire de références culturelles, références non seulement aux textes du patrimoine, mais à l'ensemble de l'héritage social inscrit dans des réseaux d'intertextualité. Un genre est également un produit de configurations linguistiques cristallisées, sédimentées et stabilisées au cours du temps. Il se présente comme un lieu pour l'apprentissage intégré de ressources langagières ; apprendre à écrire et à parler signifie mobiliser ces ressources.

Le genre oriente les dimensions à enseigner : les contraintes de la situation, les plans textuels, les unités linguistiques caractéristiques, les unités de sens, 
etc. L'élaboration d'un modèle didactique du genre (Dolz \& Schneuwly, 1998) suppose l'identification des dimensions enseignables qui peuvent générer des activités et des séquences d'enseignement. La définition de ces dimensions de manière précise facilite les possibilités de son enseignement. Dès que les objets d'enseignement sont décrits et explicités, l'entrée dans l'enseignement se voit facilitée. Ce modèle peut être également conçu comme une sorte de matrice permettant d'intégrer les nouveaux textes à des préconstruits existants. Le travail scolaire à partir du modèle du genre peut être défini comme une dynamique qui distingue les contraintes et les libertés possibles dans la production de nouveaux textes. Les contours flous et labiles du genre sont ainsi susceptibles de susciter la créativité et l'autonomie des apprenants.

Le genre permet au didacticien des langues de comprendre une série de transformations importantes. La première a trait au traitement didactique indispensable que subissent les pratiques langagières en situation scolaire (Schneuwly \& Dolz, 1997). Les genres à l'école se transforment et se dédoublent. La définition des dimensions enseignables des genres suppose une prise de distance et une rupture partielle avec les pratiques de référence pour constituer un milieu qui facilite leur appropriation. La deuxième porte sur l'ingénierie didactique, le genre est un outil dans la construction des séquences didactiques. Les connaissances sur le genre permettent l'évaluation des capacités initiales de l'apprenant, le choix d'ateliers de travail, d'activités scolaires et des supports ainsi que l'organisation de l'ensemble des modules et des activités dans une séquence didactique (Dolz, Noverraz \& Schneuwly, 2000).

\section{Les outils pour enseigner les genres : modèles et séquences didactiques}

Deux outils fondamentaux ont été élaborés et conceptualisés afin d'organiser l'enseignement de la production textuelle par les genres. Le premier est celui du modèle didactique des genres (de Pietro et Dolz, 1997 ; Dolz \& Schneuwly, 1998 ; de Pietro et Schneuwly, 2003) et concerne la formalisation des composantes enseignables des genres oraux et écrits pour l'enseignement. Le deuxième est celui de la séquence didactique (Dolz, Noverraz \& Schneuwly, 2000) et concerne la construction de démarches d'enseignement.

Un modèle didactique désigne la description provisoire des principales caractéristiques d'un genre textuel en vue de son enseignement. Le modèle didactique du genre est une élaboration en ingénierie didactique qui explicite les dimensions suivantes :

- les savoirs de référence à mobiliser pour travailler les genres ;

- la description des différentes composantes textuelles spécifiques ;

- les capacités langagières de l'élève qui les concernent.

Les modèles didactiques des genres cherchent à orienter les pratiques d'enseignement et, à cette fin, la description des caractéristiques du genre doit toujours présenter un caractère opérationnel. Un modèle didactique s'élabore en s'appuyant sur des données issues des savoirs théoriques, de l'analyse de comportements d'experts et de l'analyse de comportements d'enseignants. Ils s'élaborent de manière interactive en fonction de mises à l'épreuve successives, mais toujours en fonction de trois critères de validité didactique, c'est à dire :

- un critère de légitimité, qui suppose des ressources et des savoirs valides, 
soit en raison de leur statut académique, soit par le fait qu'ils sont considérés comme légitimes par des experts du domaine concerné ;

- un critère de pertinence, qui mesure l'adéquation des ressources et des savoirs choisis en fonction des finalités et des objectifs scolaires ;

- un critère de solidarisation, qui assure la cohérence de l'ensemble des ressources convoquées. De ce point de vue, les savoirs du modèle didactique se présentent comme un tout et prennent un sens nouveau dans le cadre de l'enseignement-apprentissage.

A titre d'exemple, le schéma suivant synthétise, sans préciser les dimensions linguistiques, les principales catégories de composantes du modèle didactique du genre textuel.

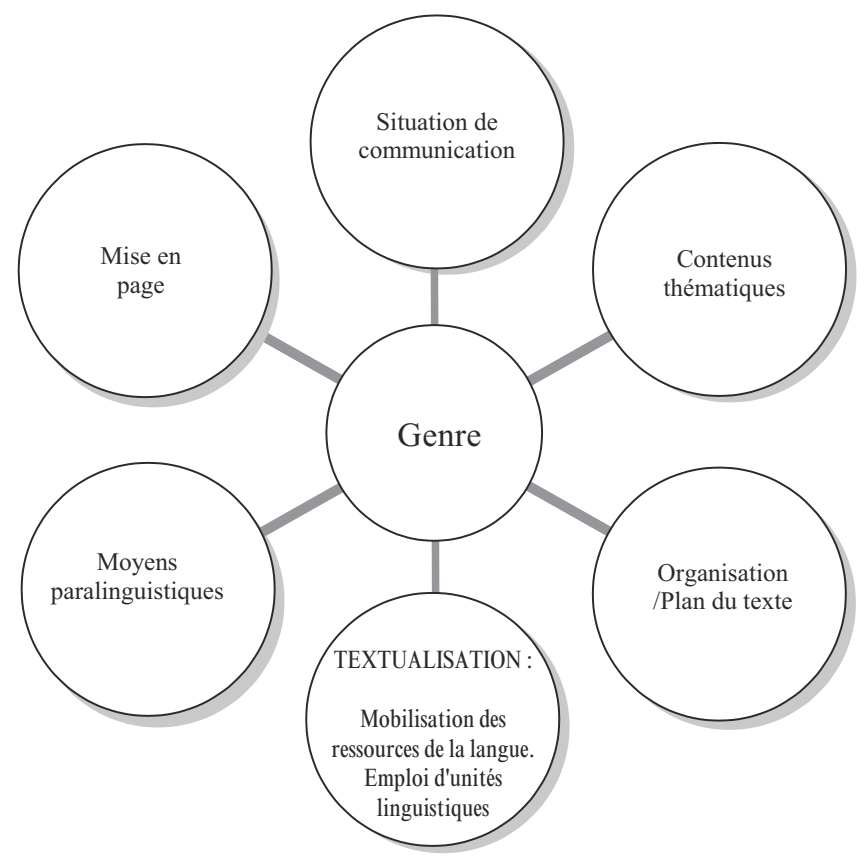

Figure 1. Modèle didactique du genre, les dimensions enseignables

Le modèle didactique du genre présente idéalement l'ensemble des ressources qui pourraient être transformées en contenus potentiels d'enseignement et mobilisées dans les activités scolaires. Il permet plusieurs réalisations, qui permettent de le considérer comme la base de données d'une démarche générative permettant la construction de tout un ensemble de séquences didactiques.

La séquence didactique se définit comme un dispositif didactique créé pour l'enseignement d'un genre textuel. Le schéma ci-dessous (page suivante) présente la structure de base d'une séquence didactique.

Dans le cadre d'un projet de communication intégrant les différentes dimensions du travail sur un genre textuel, la séquence didactique s'organise en quatre étapes : la mise en situation, la production initiale, les ateliers et la production finale.

Le projet est présenté au moment de la mise en situation. Ensuite, les élèves 


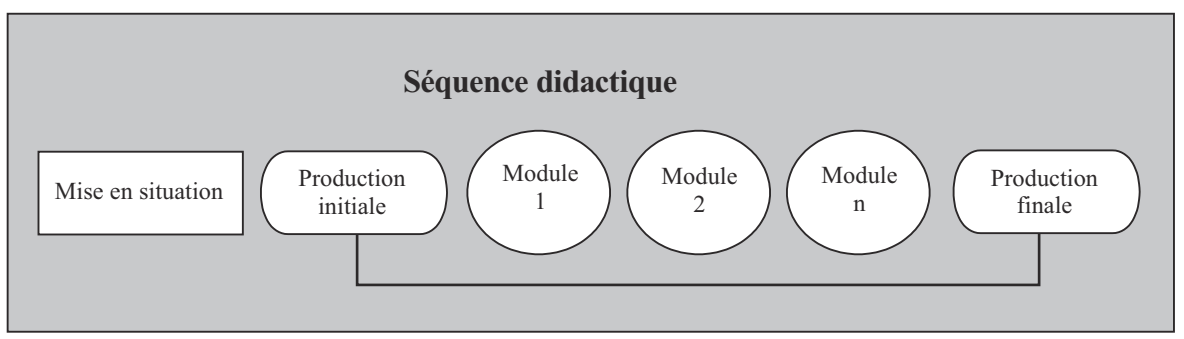

Figure 2. La séquence didactique

réalisent une première production textuelle dans le cadre du projet. Cette première production permet à l'enseignant l'observation et l'évaluation des capacités initiales des élèves de manière à cerner les principales difficultés qu'ils rencontrent par rapport à la production d'un genre textuel.

Une série d'ateliers composés de diverses activités scolaires et d'exercices constitue la troisième étape. Chaque atelier est destiné à améliorer une des composantes du texte à rédiger ou à résoudre un problème d'écriture observée dans les productions initiales des élèves. Les ateliers visent à combler les difficultés apparues lors de la première production et touchent aux quatre niveaux de l'activité langagière : le contexte de production, les contenus, l'organisation du texte et les unités linguistiques qui assurent la connexion et la cohésion du texte. Les ateliers sont par ailleurs conçus comme des modules qui peuvent s'adapter à divers contextes scolaires et à des groupes d'apprenants hétérogènes.

La quatrième et dernière étape prévoit la réalisation d'une production finale dans laquelle les élèves mettent en pratique les savoirs et savoir-faire appris et exercés dans les ateliers. La production finale est le lieu par excellence de l'intégration des apprentissages. Pour permettre l'évaluation formative ou certificative critériée de la production finale, des supports sous forme de grilles sont présentés.

Le dispositif séquence didactique est présenté non seulement comme un outil de travail scolaire au service de la production orale et écrite, mais aussi comme un outil permettant une meilleure compréhension de ce qui fait obstacle à l'oral et l'écriture de manière à favoriser un contrôle conscient des processus impliqués dans la production orale ou dans la rédaction d'un texte.

\section{De l'improvisation théâtrale à la saynète en passant par le canevas}

Cette dernière partie vise à présenter un exemple de l'exploitation des genres dans l'enseignement-apprentissage de la production textuelle. L'exemple que nous avons choisi, toutefois, est intentionnellement atypique, puisqu'il fait appel à un genre difficilement délimitable, l'improvisation théâtrale. Nous tenterons de l'appréhender ici comme un genre d'activité orale qui implique une diversité de genres textuels. Texte oral improvisé, appartenant peut-être davantage aux genres premiers qu'aux genres seconds, l'improvisation théâtrale s'avère être un outil judicieux pour l'apprentissage de l'écriture dramaturgique, en ce qu'elle donne accès à l'élève aux formes d'écrit intermédiaires nécessaires à la production d'un genre textuel écrit : la saynète. 


\section{L'improvisation théâtrale : un genre textuel oral?}

L'improvisation, entendue comme mode d'expression dramatique exercé spontanément devant public, existerait depuis toujours. Il nous est tous déjà arrivé, à un moment ou un autre de notre existence, de volontairement profiter du rire que l'on avait provoqué chez les autres pour en mettre un peu plus. Quiconque n'a jamais joué un peu la comédie, histoire d'alléger son existence ou celle d'autrui, d'augmenter son pouvoir de persuasion ou d'en arriver à ses fins. Il nous faut alors préciser ce que nous entendons par « mode d'expression dramatique exercé spontanément devant public» : il faut, entre autres, postposer 1'adjectif « volontaire » à « public », mais surtout il nous faut comprendre ce qui fait la spécificité de l'improvisation théâtrale par rapport au théâtre, genre auquel elle est étroitement associée. Tentons de retracer les transformations de cette forme théâtrale.

Les inspirations les plus anciennes de l'improvisation théâtrale sont les atellanes, un genre théâtral latin du III ${ }^{\mathrm{e}}$ siècle, lui-même inspiré de la Comédie Nouvelle grecque, comédie de mœurs à intrigue simple qui réserve une importante place à l'expression des sentiments. L'Atellane reprend les costumes et les masques de la Comédie Nouvelle grecque et les caricature : quatre personnages masqués improvisent sur la base d'un canevas. Ces farces latines, prémices de la comédie italienne, inspireront les personnages de Pantalon, de Polichinelle et du Docteur de la Commedia dell'arte.

Troupes d'amateurs cultivés puis de professionnels, les comédiens italiens jouent au $16^{\mathrm{e}}$ siècle dans les petites cours italiennes un théâtre en partie improvisé. Réunissant dans un seul spectacle les types populaires masqués de plusieurs régions de l'Italie, les acteurs de la Commedia dell'arte allient parfaitement la bouffonnerie au romanesque, les traditions populaires à la culture antique (Attinger, 1950). Ils improvisent sur la base d'un canevas. Leur jeu, sorte de « langage énergique de la scène se base sur une déformation consciente et intelligente du corps » (Taviani \& Schino, 1984, p. 47), c'est ce qui d'ailleurs assurera la pérennité de cette forme théâtrale. À son achèvement, la comédie italienne compte une dizaine de personnages et son répertoire va de la grosse farce à la tragicomédie. Ses premières influences ont été celles d'acteurs sur des acteurs. Les farceurs de la première moitié du $17^{\mathrm{e}} \mathrm{s}$ 'en sont inspirés, Molière la fera entrer dans la littérature : «Il est sans doute le premier des écrivains français à l'avoir comprise par la base, c'est-à-dire à partir du jeu ; l'acteur chez Molière a renseigné l'auteur » (Attinger, 1950, p. 9). Aussi, pour les acteurs italiens comme pour Molière, l'improvisation appartient à l'action et à l'art indissociable de l'acteur (Tournier, 2003).

La résurgence de l'improvisation théâtrale, disparue avec le déclin de la Commedia dell'arte, est associée à la figure de Constantin Stanislavski. Le metteur en scène russe l'utilise comme fondement de l'apprentissage du métier de comédien : l'improvisation permet au comédien de revivre des sentiments éprouvés. Cette vision tranche avec celle de Jacques Lecoq. Pour le réputé pédagogue du théâtre, l'improvisation est ce qui conduit le comédien à retrouver la page blanche de toute chose, à se démunir de son savoir et de ses représentations pour pouvoir se rendre disponible à tout ce qui pourra traverser le corps et s'exprimer à travers lui (LeCoq, 1987). Les idées des deux maitres se rejoignent quant à l'utilisation de l'improvisation comme moteur de la création. Des metteurs en scène 
tels que Meyerhold, Gordon Craig, Jacques Copeau, Charles Dullin s'intéressent à la comédie improvisée (Tournier, 2003). La vision de Stanislavski de la formation de l'acteur sera reprise aux États-Unis avec la création du très célèbre Actors Studio par Elia Kazan en 1947. Dès le $20^{\mathrm{e}}$ siècle, partout dans le monde, l'improvisation est devenue une forme d'exploration théâtrale incontournable.

A l'heure actuelle, l'improvisation théâtrale se pratique un peu partout dans le monde. Elle sert à de plus en plus de protagonistes et se pratique dans une multitude de lieux. Son succès s'explique facilement : la pratique de l'improvisation contribue, entre autres, à réagir, en développant par exemple le sens de la répartie, à prendre sa place, à être présent et disponible, à entrer en relation avec l'autre et avec l'espace, à s'exprimer par le corps, à s'adapter à la situation de communication, à s'accepter et à accepter l'autre, à se décentrer, à s'engager et à engager l'autre. Représentation théâtrale, elle prend la forme de matchs où, selon les directives d'un maitre de jeu, des jouteurs créent des situations en simultané sous l'œil attentif d'un public qui, parfois, exprime son appréciation, en plus de ses applaudissements, par un vote. Technique de communication, elle est cette valeur ajoutée à l'employé du secteur tertiaire. A l'école, que ce soit dans le scolaire ou le parascolaire, elle est un exercice nécessaire à l'apprentissage du jeu dramatique. Elle donne accès aux élèves à une « analyse de la réalité à partir d'un discours donné dans un langage artistique original», elle est un « moyen d'interrogation et de vérification de la communicabilité du discours tenu », elle est aussi un « comportement ludiquement élaboré à l'intérieur d'une situation » (Ryngaert, 1996, pp. 8-9). Elle profite du pouvoir d'attraction du jeu, en ayant comme caractéristique fondamentale de se fonder sur un va-et-vient entre la conscience des règles du jeu et l'oubli de ces règles dans une démarche simultanée (Ryngaert, 1996). L'improvisation théâtrale autorise les essais, les reprises et les retours en arrière ; elle rend possible les allers-retours propres à l'écrit.

Peut-on pour autant parler de genre? Si on reprend les trois dimensions essentielles du genre, à savoir des contenus dicibles, une structure communicative particulière des textes et des configurations spécifiques d'unités langagières, peut-on caractériser l'improvisation théâtrale ? Lieu de toutes les possibilités, l'improvisation se joue à la manière d'un film de cinéma muet, d'une comédie musicale, d'un péplum, d'un polar, d'un roman de Jules Verne, d'une pièce de Ionesco, d'un film de Fellini et de tout ce qui appartient aux jouteurs impliqués. La multiplicité des contenus est donc une de ses spécificités : il y a les règles et les conventions du jeu, mais il y a aussi leur oubli. Elle se joue seul ou à plusieurs, avec ou sans directives de départ; sa structure communicative est donc monologuée ou dialoguée et se planifie parfois au préalable, mais toujours en simultané. Cette structure se module en fonction des jouteurs, en fonction du style imposé, s'il en est un, et en fonction du temps imparti et des réactions du public. Cette variété, ce réservoir de possibles se rapportent aussi à la textualisation. L'improvisation se distingue donc du théâtre par cette création simultanée qui permet une écriture qui intègre directement les réactions du public, mais elle ne saurait présenter des formes stabilisées. Il s'agit donc d'un genre d'activité orale qui implique une diversité de genres textuels. Pour nous, elle est un genre multigenres et c'est ce caractère évolutif que nous voulons mettre à profit pour l'enseignementapprentissage de la production écrite. 


\section{D'un genre premier à un genre second ; l'écrit et l'oral intermédiaire comme outils dans une séquence d'enseignement sur la production écrite d'une saynète}

Dans l'idée de répondre à la nécessité d'organiser des dialogues multiples entre les élèves autour de l'écriture (Seweryn \& Bucheton, 1999), nous croyons que l'écriture d'une saynète est un choix judicieux, puisqu'elle permet de mettre à la disposition des élèves des genres d'oral et d'écrit intermédiaires, l'improvisation et le canevas. Ces outils, d'une part, mettent en œuvre le dialogisme présent dans tout texte, soit la prise en charge et la hiérarchisation des différentes voix dans le discours. D'autre part, ils donnent à voir la manière dont se réorganisent les compétences internes, ce que Alcorta (2001) en traitant du brouillon appelle la «construction de l'architecture mentale du scripteur » (p. 131). Ils sont des outils structurant le processus d'écrire. Par des occurrences fréquentes, l'apprenti scripteur fait progressivement bouger ses postures d'écriture. L'écrit et l'oral intermédiaires sont donc conçus comme des outils de construction de texte, des moyens de gérer la production (Alcorta, 2001).

De façon concrète, l'enseignant qui veut faire apprendre l'écriture dramaturgique à ses élèves peut procéder, comme le font de nombreux collectifs artistiques, en les faisant improviser par petits groupes sur la base d'un thème. Après ces productions initiales, les groupes annotent les idées qui leur semblent intéressantes pour une éventuelle saynète. De cette façon, des premiers échanges fictifs sont créés et, même si beaucoup d'éléments ne seront pas repris, des idées ont germé. Ensuite, à partir de ces improvisations, les élèves sont davantage en mesure de s'entendre sur le canevas de la saynète. L'enseignant peut choisit d'intervenir peu ou beaucoup dans le cadrage de ces improvisations ; devant un groupe indiscipliné ou peu productif, il lui faudra peut-être resserrer le jeu, mais, avec certains élèves, son rôle sera de guider, d'aider à aller plus loin.

L'étape suivante consiste en la rédaction du canevas par les élèves, soit le squelette de la saynète, ses grandes lignes. Pour l'enseignant, il s'agit de faire travailler l'élève sur la mise en intrigue, c'est-à-dire la sélection et l'agencement des évènements de manière à ce qu'ils forment un tout, une histoire, une action complète :

Suivre une histoire, c'est avancer au milieu de contingences et de péripéties sous la conduite d'une attente qui trouve son accomplissement dans la conclusion [...] Elle donne à l'histoire un « point final », lequel, à son tour, fournit le point de vue où l'histoire peut-être aperçue comme formant un tout (Ricœur, 1983, p. 103).

À partir des notes qu'ils auront prises des premières improvisations, à partir aussi des échanges rétrospectifs qu'ils auront en équipe, chaque équipe doit rédiger le canevas de la saynète. Des improvisations plus ciblées peuvent être faites en vue de débloquer certains « nœuds» dans l'élaboration de la trame.

Une fois le canevas rédigé, il peut être intéressant de travailler la mise en texte en regardant les transformations nécessaires des dialogues, de manière à passer d'une production orale spontanée à un écrit oralisé. Ici, l'utilisation des transcriptions de passages d'improvisation s'avère fort pertinente. La compréhension et la rédaction des indications scéniques, les didascalies internes (intégrées aux dialogues, elles caractérisent la situation d'énonciation) et externes (les indications données en marge du dialogue), pourraient faire l'objet d'un module. 
Il y a aussi, tout au long de la séquence d'enseignement, à donner à lire des saynètes pour favoriser la reconnaissance de la structure du genre, laquelle, nous l'avons dit, est extrêmement variable.

L'étape finale est le moment de la rédaction de la saynète. Les différentes étapes intermédiaires devraient normalement permettre à ce qu'ensemble, les équipes puissent mettre en mots des répliques de théâtre, lesquelles s'organisent dans des dialogues, et que le tout forme une entité cohérente, le fruit d'une création collective.

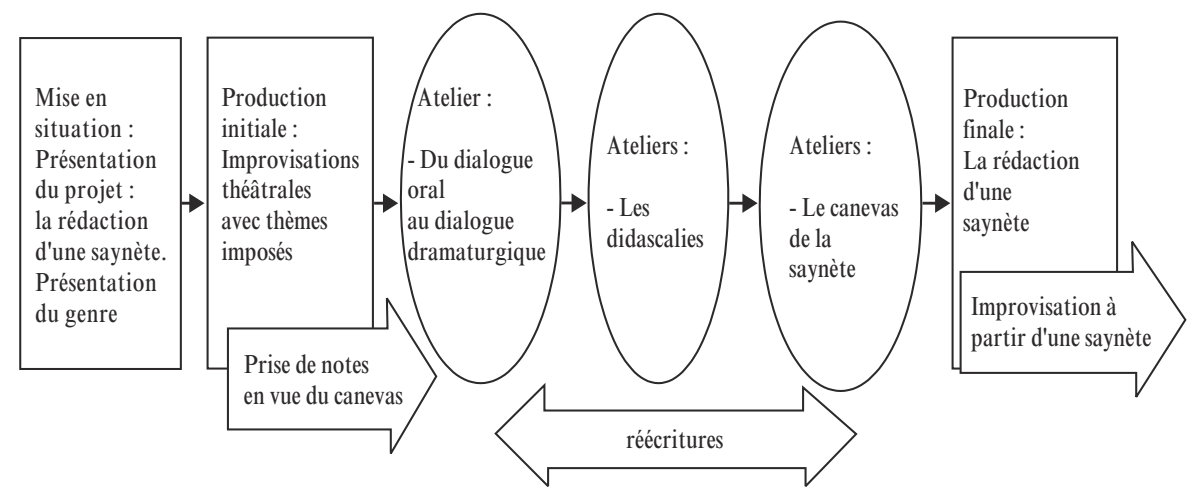

Figure 3. Schématisation d'une séquence didactique pour travailler la saynète

\section{Conclusion}

La présentation des genres textuels comme unité pour l'enseignement-apprentissage de la production orale et écrite nous permet maintenant de répondre aux questions soulevées par Halté (2005).

L'oral peut-il devenir un objet d'enseignement ? Cette contribution a été l'occasion pour nous de confirmer l'intérêt en didactique d'une entrée par les genres textuels et de montrer ses principaux apports pour l'enseignement-apprentissage de la production orale et écrite. Plusieurs arguments sont venus étayer notre point de vue, nous en reprenons les principaux. D'abord, le genre constitue une unité de travail qui donne du sens à l'activité d'enseignement-apprentissage, puisqu'il fournit un horizon d'attentes aux apprenants. Ensuite, le genre permet d'articuler les pratiques sociales de référence - dans notre exemple, celle des artisans du théâtre - et les objets scolaires. Cette transformation d'un genre social à un genre scolaire suppose une délimitation des dimensions enseignables du genre. Son analyse à priori vient d'ailleurs clarifier les objets d'enseignement. Dans ce cadre, l'école est considérée comme un authentique lieu de communication, les situations scolaires constituent des occasions de réception et de production des textes (Schneuwly \& Dolz, 1997). L'école transforme les genres. En didactique, la prise en compte de l'intertextualité est importante puisqu'elle permet de mettre l'élève en situation de dialogue avec les textes qui le précèdent. C'est avec ce " déjà-là », qu'il s'approprie la textualité et intègre le dialogisme dans ses propres productions. Les travaux de notre équipe montrent que cet « Objet Verbal Mal Identifié » (Halté, 2005, p. 12) peut devenir un objet relativement 
précis dans le cadre d'une didactique des genres formels oraux, intégrant très précisément des objectifs sur différentes dimensions multimodales de l'oral (ressources de la langue, timbre, intonation, débit, rythme, rapport avec le corps, postures, etc.). Cette didactique n'est pas en contradiction avec d'autres entrées possibles, didactiques (voir pour une synthèse voir Nonnon, 1999 ; Garcia-Debanc \& Plane, 2004 ; Delcambre \& Garcia-Debanc, 2001/2002) et pédagogiques (libération et gestion de la parole en classe, parler pour apprendre, oral spontané, etc.). Ce qui nous semble important de souligner c'est que l'oral, grâce aux nouvelles propositions didactiques sur les genres, n'est plus un seul objet d'invocation, mais dispose désormais d'une série d'outils concrets d'enseignement dont les retombées sur les apprentissages sont positives.

Quelles sont les possibilités d'articulation de l'oral et de l'écrit? Nous focalisant sur l'improvisation théâtrale, qui est un genre d'activité langagière issu des pratiques théâtrales de référence, nous avons voulu montrer ses potentialités comme outil didactique. Genre multigenres, elle possède les caractéristiques générales du jeu dramatique, mais se définit aussi par des propriétés uniques et spécifiques : elle se crée immédiatement devant un public, elle combine une variété de styles, elle est orchestrée par un maître de jeu et autorise un dialogue particulier avec le public. Nous inspirant des pratiques de référence de certains collectifs d'auteurs dramaturgiques, nous avons illustré comment, dans un processus d'allers-retours, elle peut servir la rédaction d'un canevas et d'une saynète. Ce faisant, il nous a été possible de mettre à profit la dynamique intrinsèque aux genres textuels, à la fois modèles communs nés de régularités dans l'usage et véhicules des transformations issues des situations communicatives changeantes. Passant d'un genre oral atypique, genre premier, pour aller vers un genre second écrit, la saynète, nous avons voulu prouver l'affirmation de Schneuwly comme quoi l'apparition des genres seconds chez l'enfant n'est pas le point d'aboutissement, mais le point de départ d'un long processus de restructuration, qui, à terme, va produire une révolution de ses capacités langagières. Ainsi, le travail sur la saynète permet d'articuler l'écrit et l'oral dans le cadre du jeu dramatique. Ici, nos positions coïncident avec celles d'Halté (2005). Le slogan évoqué par ce dernier, "l'oral et l'écrit, un seul et même combat » (p. 26), souligne l'importance que l'auteur attribue au développement des activités langagières orales et écrites. En effet, nous pensons avoir pu exemplifier les potentialités d'une didactique intégrée de la réception et de la production orale et écrite. Il est vrai, comme le dit Halté, que «l'oral en français servira non seulement au développement des compétences orales mais aussi au développement de l'écrit » (p. 28). Mais les formes d'articulation et d'interaction entre l'oral et l'écrit sont diverses et plus complexes : si l'oral sert à l'écrit le contraire est aussi vrai. Le travail sur les genres permet, d'une part, un travail autonome sur l'oral et sur l'écrit et, d'autre part, des formes diverses d'intertextualité. Ainsi, nous avons montré ailleurs que le genre «communication affichée » ou « poster» pouvait être un outil au service de l'exposé oral (Dolz, Hanselman \& Ley, 2006 ; Pfeiffer, Demaurex \& Dolz, 2007). Les formes d'aller-retour entre l'oral et l'écrit sont diverses. Nonnon (2002) a montré les possibilités didactiques des passages entre les notations et l'oralisation. L'intégration de l'oral dans l'enseignement, en plus de renforcer l'enseignement de l'écrit, permet de mieux saisir dans les pratiques les interactions au service du développement des capacités langagières des apprenants. 
Comment articuler l'oral objet à enseigner et l'oral outil pour apprendre? La question est complexe et nous ne saurions mettre fin au débat. Nous croyons toutefois qu'une définition trop large de l'oral empêche sa délimitation en tant qu'objet : si à chaque fois que l'enseignant ou les élèves ouvrent la bouche, ils font de l'oral, à la fois tout et rien devient oral. Certains ont reproché à notre entrée par les genres formels d'être une entrée trop étroite ; c'est ne pas tenir compte de la vision dynamique et ouverte des genres. Nous avons fait le choix de nous focaliser surtout sur l'enseignement de la parole publique, sans nier d'autres entrées possibles sur les genres de la vie quotidienne, notamment avec les plus jeunes élèves (description d'itinéraires, rituels, jeux de rôles). L'intérêt de la modélisation didactique sur des objets d'enseignement bien délimités est de permettre de pointer les dimensions enseignables et celles qui posent problème aux apprenants. Un objet d'enseignement-apprentissage bien délimité facilite l'élaboration de critères d'évaluation. Une didactique intégrée des langues met en évidence les interactions entre les différentes activités langagières à développer: le lire, l'écrire, le parler, l'écouter et l'interagir. Chacune de ces activités constitue un outil pour les autres. Les genres, comme nous l'avons montré, sont à la fois objet d'enseignement et outil d'apprentissage pour le français et pour les autres disciplines scolaires.

Le débat, entrepris par Halté dans les années 1992, a fait avancer la didactique du français. Beaucoup reste à faire, disait-il en 2005, mais les voies ouvertes pour développer le langage oral et écrit, depuis lors, sont prometteuses et méritent d'être développées.

\section{Références bibliographiques}

ADAM, J.-M. (1992) : Les textes, types et prototypes. Paris : Nathan.

AlCORTA, M. (2001) : «Une approche vygotskienne du développementdes capacités d'écrit; Le brouillon : un outil pour écrire », in Brossard, M. \& Fijalkov, J., Apprendre à l'école : perspectives piagétiennes et vygotskiennes, Bordeaux : Presses universitaires de Bordeaux, 123-151.

A tTiger, G. (1950) : L'esprit de la Commedia dell'Arte dans le théâtre français. Neufchâtel : A la Baconnière.

BAKhtine, M. (1934/1978) : Esthétique et théorie du roman. Paris : Gallimard. - (1984) : Esthétique de la création verbale. Paris : Gallimard.

BOTA, C. \& BRONCKART, J.-P. (2007) (2008) : «Volochinov et Bakhtine : deux approches radicalement opposées des genres de texte et de leur statut », in: Linx, 56, 67-83.

BRONCKART, J.-P. (1996) : Activité langagière, textes et discours. Pour un interactionisme socio-discursif. Neuchâtel-Paris : Delachaux et Niestlé.

BROSSARD, M. (2001) : «Approche socio-historique des situations d'apprentissage de l'écrit », in Brossard, M. \& Fijalkov, J., Apprendre à l'école : perspectives piagétiennes et vygotskiennes, Bordeaux : Presses universitaires de Bordeaux, 51-80

CANVAT, K. (1999) : Enseigner la littérature par les genres ; Pour une approche 
théorique et didactique de la notion de genre littéraire. Bruxelles : Deboeck/Duculot.

Chareaudeau, P. \& Maingueneau, D. (2001) : Dictionnaire d'analyse du discours. Paris : Seuil.

Combe, D. (1992) : Les genres littéraires. Paris : Hachette.

De Pietro, J.-F. \& Dolz, J. (1997) : «L'oral comme texte : comment construire un objet enseignable? », Éducation et recherches, 19/3, 335-359.

De Pietro, J.-F. \& SChNeuWly, B. (2003) : « Le modèle didactique du genre : un concept de l'ingénierie didactique », Les Cahiers Théodile, 3, 27-52.

DolZ, J. HANSELMANN, S. \& LEY, V. (2006) : «La communication affichée au service de l'exposé oral : apprentissage de l'usage de l'écrit comme support de la prise de parole en public », in : SchneuwlyB. \& Thévenaz-Christen T. (Ed.) Analyses d'objets enseignés, Bruxelles : De Boeck, 143-157.

DolZ J. \& SCHNEUWLY, B. (1996) : «Genres et progression en expression écrite : éléments de réflexion à propos d'une expérience romande », Enjeux, 37/38, 49-75.

Dolz, J. \& SCHNEUWLY, B. (1998) : Pour un enseignement de l'oral ; Initiation aux genres formels à l'école. Paris : ESF éditeur.

Dolz, J., Noverraz, M. \& Schneuwly, B. (2001) : Séquences didactiques pour l'oral et pour l'écrit; Notes méthodologiques. Bruxelles : De Boeck.

Dolz, J., Moro, C. \& Pollo, A. (2001) : «Le débat régulé : de quelques outils et de leurs usages dans l'apprentissage », Repères, 22, 76-95.

Dolz, J., S ChneuWly, B., \& ThÉVEnAZ-Christen, T. (2007) : « Au fondement de la didactique : l'articulation vygotskienne de l'objet enseigné à l'outil médiateur », Actes du colloque d'Albi sur Vygotski et les recherches en éducation et en didactique des disciplines, 23-24 avril 2007.

FOWLER, A. (1982): Kinds of Literature. An introduction to the theory of genres and modes. Harvard University Press : Cambridge.

GARCiA-Debanc, C. \& Delcambre, I. (Eds). Enseigner l'oral. Repères, 24/25, 2001-2002.

GARCIA-DEBAnC, C. \& Plane, S. (Ed.), Comment enseigner l'oral à l'école primaire?, Paris : Hatier, 263-310

Genette, G. (1979) : Introduction à l'architexte. Paris : Seuil.

Halté, J.-F. (1992) : La didactique du français. Paris : Presses universitaires de France (coll. Que sais-je?)

— (Ed.) (1993) : Inter-actions. Casum : Université de Metz, Metz.

HALté, J.-F. \& RisPAiL, M. (Eds) (2005) : L'oral dans la classe ; compétences, enseignement, activités. Paris : L'Harmattan.

JAUSS, H. J. (1975/1978) : Pour une esthétique de la réception. Paris : Gallimard.

Johnstone, K. (1989) : Impro : Improvisation and the Theatre. Londres : Paperback.

LaVergne, J.-M. \& Gravel, R (1987) : Impro I, II, Montréal, Leméac.

LECOQ, J. (1987) : Le théâtre du Geste, mimes et acteurs. Paris : Bordas.

LOICQ-Berger, M.-P. (1980) : "Pour une lecture du roman grec : son intérêt pluriel, ses prolongements », Les Études classiques, $t$. 48, Faculté universitaire de Namur, 23-42.

NONNON, É. (1999) : «L'enseignement de l'oral et les interactions verbales en classe : champs de référence et problématiques », Note de synthèse», Revue 
française de pédagogie, 129, 87-131.

— (2002) : «Des interactions entre oral et écrit : notes, traces écrites et leurs usages dans la pratique orale », Pratiques, 115-116, 73-92.

Plane, S. \& SCHNEUWLY, B. (2001) : «Regards sur les outils de l'enseignement du français. Un premier repérage », Repères, 22, 3-18.

Pfeiffer-Ryter, V., Demaurex, M. \& Dolz, J. (2007) : «Prendre des notes pour un exposé oral : intégrer lecture, écriture et expression orale au primaire », in Falardeau, E., Fisher, C., Simard, C. \& Sorin, N. La didactique du français : les voies actuelles de la recherche, Québec : PUL, 235-256.

RICEUR, P. (1991) : Temps et récit, l'intrigue et le récit historique. T. 1, Paris : Seuil.

RYNGAERT, Jean-Pierre, (1996) : Le jeu dramatique en milieu scolaire. Bruxelles : De Boeck.

SCHAEFfer, J.-M., (1989) : Qu'est-ce qu'un genre littéraire? Paris : Seuil. - (2001) : «Genres littéraires », in Dictionnaire des genres et notions littéraires (pp. 353-358), Encyclopaedia Universalis. Paris : Albin Michel.

SCHNEUWLY, B. \& DOLZ, J. (1997) : «Les genres scolaires; des pratiques langagières aux objets d'enseignement », Repères, 15, 27-40.

SCHNEUWLY, B. (1994) : "Genres et types de discours : considérations psychologiques et ontogénétiques ", in Reuter, Y., Les interactions lecture-écriture. Berne : Peter Lang.

SEWERYN, B. \& BUCHETON, D. (1999) : «Le carnet d'écrivain : d'un grand nombre d'écrits vint la créativité ! ", Le français aujourd'hui, 127, 73-82.

TAViAni, F. \& SCHIMO, M. (1984) : Le secret de la Commedia dell'Arte, la mémoire des compagnies italiennes au 16 $6^{e}, 17^{e}, 18^{e}$ siècles. Paris : Bouffonneries.

TOdorov, T. (1978) : Genres du discours. Paris : Seuil.

- (1979/1984) : Préface, in Esthétique de la création verbale, Paris : Gallimard, 7-23

Toulou, S. (2005) : « La fomation des griots : quelle forme éducative ? », in : O. Maulini \& C. Montandon (Ed.), Les formes de l'éducation : variétés et variations, Bruxelles : De Boeck, 83-102

TOURNIER, C. (2003) : Manuel d'improvisation théâtrale. Genève : Éditions de l'eau vive.

VyGOTSKY, L. S. (1933/1985) : «Les bases épistémologiques de la psychologie », in Schneuwly, B. \& Bronckart, J.-P. (dir.), Vygotsky aujourd'hui. Paris : Delachaux et Niestlé.

— (1933/1985) : «La méthode instrumentale en psychologie», in Schneuwly, B. \& Bronckart, J.-P. (dir.), Vygotsky aujourd'hui. Paris : Delachaux et Niestlé.

— $(1933 / 1985)$ : «Le problème de l'enseignement et du développement mental à l'âge scolaire », in Schneuwly, B. \& Bronckart, J.-P. (dir.), Vygotsky aujourd'hui. Paris : Delachaux et Niestlé.

- (1934/1997) : Pensée et langage. Paris : La Dispute.

WIRTHNER, M. (2006) : La transformation de pratiques d'enseignement par l'outil de travail, Observation de séquences d'enseignement du résumé écrit de texte informatif à l'école secondaire. Thèse de doctorat en Sciences de l'éducation, Université de Genève (non publiée). 